\title{
REPORTE DE CASOS
}

\section{Insuficiencia suprarrenal en paciente con dificultad respiratoria y úlceras en miembros inferiores}

\section{Suprarrenal insufficiency in patient with respiratory difficulty and ulcers in lower members}

Aldama Caballero, Arnaldo'; Centurión Medina, Ingrid ${ }^{1}$; Fornerón Viera, Laura1

${ }^{1}$ Ministerio de Salud Pública y Bienestar Social, Hospital Nacional de Itaugua, Servicio de Dermatología. Itaugua - Paraguay

\section{RESUMEN}

La Insuficiencia Suprarrenal es un cuadro grave que presenta numerosas manifestaciones clínicas secundarias a la depresión de la producción hormonal de dicha glándula. Las causas son varias e incluso depende de las regiones y épocas. En América Latina se debe considerar al Paracoccidioides como probable causa. Presentamos el caso de un hombre campesino, ingresado al Hospital por Insuficiencia Suprarrenal, con dificultad respiratoria y lesiones ulcerosas en piernas debidas a paracoccidioidomicosis, que también fue la causa del trastorno respiratorio y posiblemente suprarrenal.

Palabras clave: Insuficiencia suprarrenal, Enfermedad de Addison, Paracoccidioidomicosis. 


\section{ABSTRACT}

Adrenal insufficiency is a serious condition that presents numerous clinical manifestations secondary to the depression of the hormonal production of said gland. The causes are several and even depends on the regions and times. In Latin America, Paracoccidioides must be considered as a probable cause. We present the case of a peasant man, admitted to the Hospital for Adrenal Insufficiency, with respiratory distress and ulcerous leg injuries due to paracoccidioidomycosis, which was also the cause of the respiratory and possibly adrenal disorder.

Key words: Adrenal insufficiency, Addison's disease, Paracoccidioidomycosis.

\section{INTRODUCCION}

La Insuficiencia Suprarrenal (IS) es un trastorno grave, provocado por la deficiencia de hormonas producidas por las glándulas suprarrenales, principalmente los glucocorticoides, pero también los mineralocorticoides y andrógenos (1).

El trastorno puede ser primario, debido a lesión de la misma glándula, también llamada enfermedad de Addison; secundario o terciario, por alteración del eje hipotálamo-hipofisario $(1,2)$.

La causa más importante de IS primaria en Europa durante la primera mitad del siglo $X X$ fue la tuberculosis, pero en la actualidad son las enfermedades autoinmunes y neoplásicas. En niños la causa más importante es genética( $1,2,3)$.

En los países en vías de desarrollo, además de la tuberculosis y otras micobacterias, las micosis profundas producen IS primaria, más frecuentemente la histoplasmosis. En países endémicos de paracoccidioidomicosis, ésta es una probable causa $(1,2,4)$.

Las principales manifestaciones clínicas de la IS son debilidad, fatiga, anorexia, pérdida de peso, dolor abdominal, hipotensión, hiperpigmentación cutánea $(1,2)$.

Presentamos el caso de un paciente con IS primaria, afectación pulmonar y lesiones en la piel producidas por paracoccidioidomicosis.

\section{CASO CLINICO}

Paciente de 65 años de edad, agricultor, procedente de Paso Barreto (Departamento de Concepción), conocido etilista y tabaquista, internado en el servicio de Clínica Médica por mal estado general, astenia marcada, somnolencia, hipoglicemia, hipotensión, fiebre, tos, expectoración abundante y dificultad respiratoria.

El cuadro, apoyado en los estudios laboratoriales, es interpretado como IS acompañada de neumopatía probablemente bacteriana, por lo que se inicia tratamiento con hidrocortisona y antibióticos, con favorable respuesta.

Remitido en interconsulta al Servicio de Dermatología por úlceras dolorosas en ambos miembros inferiores de 1 año de evolución.

Al examen general llamaba la atención la hiperpigmentación generalizada de la piel y de la mucosa oral (Figura 1). PA: $100 / 60 \mathrm{~mm} \mathrm{Hg}$, FC: 78 lat $/ \mathrm{min}$

Figura 1. Hiperpigmentación de piel, labios y mucosa oral.

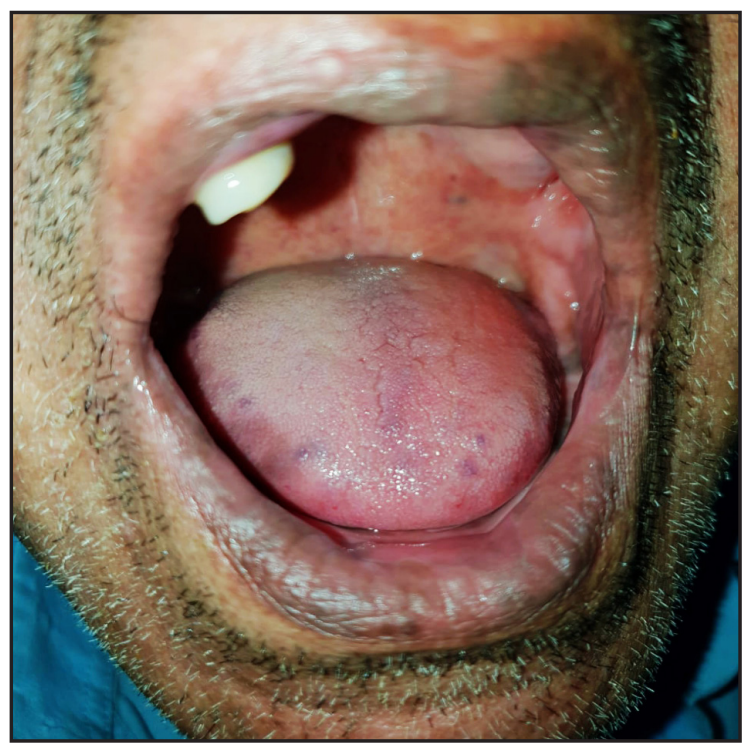


En el dorso del pie derecho presenta dos lesiones ulcerosas de $2 \times 2 \mathrm{~cm}$ aproximadamente de diámetro, de bordes elevados, bien definidos, con fondo granulomatoso, cubierto por una membrana blanquecina. Hiperpigmentación mas intensa en

Figura 2. Lesiones ulcerosas en pie derecho Hiperpigmentación.

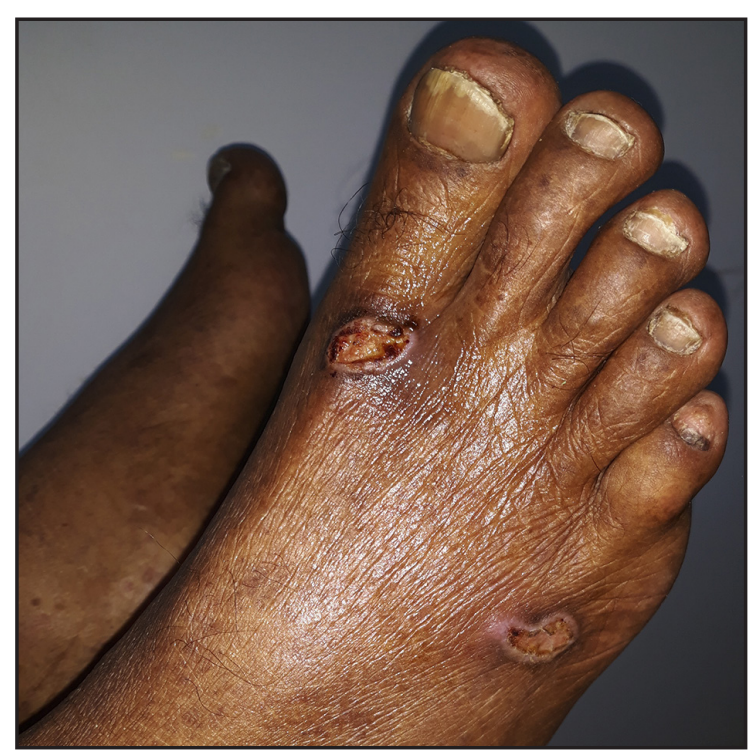

La anatomía patológica de una de las úlceras del dorso del pie informa en dermis papilar y reticular un denso infiltrado inflamatorio granulomatoso constituido por linfocitos, histiocitos y células gigantes multinucleadas de tipo reacción a cuerpo extraño, así como numerosos neutrófilos que conforman microabscesos. Llama la atención la presencia zona perilesional (Figura 2). En ambas plantas de pies además de la hiperpigmentación, múltiples pápulas de superficie queratósica, y una lesión ulcerosa en base del hallux derecho, con fondo sucio (Figura 3).

Figura 3. Hiperpigmentación, lesiones papulo queratósicas y úlcera en hallux de planta de pie derecho.

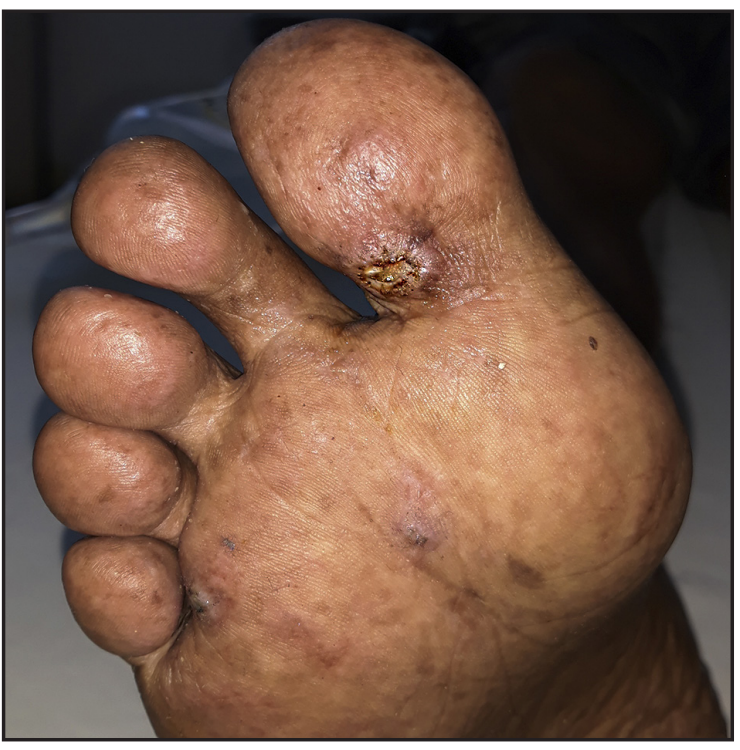

de numerosos elementos fúngicos en forma de levaduras que miden entre 10 a 20 micras de diámetro, muchos de ellos en el citoplasma de células gigantes. Se concluye tratarse de una micosis profunda a identificar por estudios micológicos (Figura 4).

Figura 4. Anatomia patológica. H.E. denso infiltrado inflamatorio con múltiples elementos micóticos.

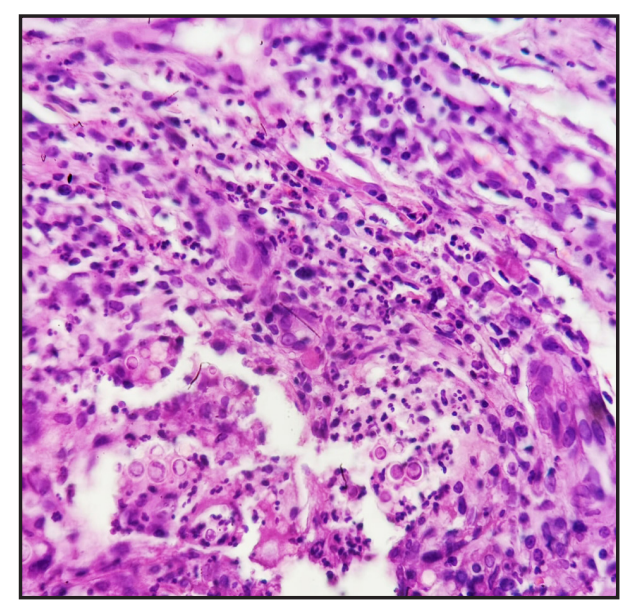


En el examen micológico directo, de la piel y esputo se observa: elementos levaduriformes multibrotantes compatibles con Paracoccidioides spp (Figura 5). El cultivo

Figura 5. Examen micológico directo. Se observan múltiples elementos micóticos multibrotantes. Paracoccidioides spp

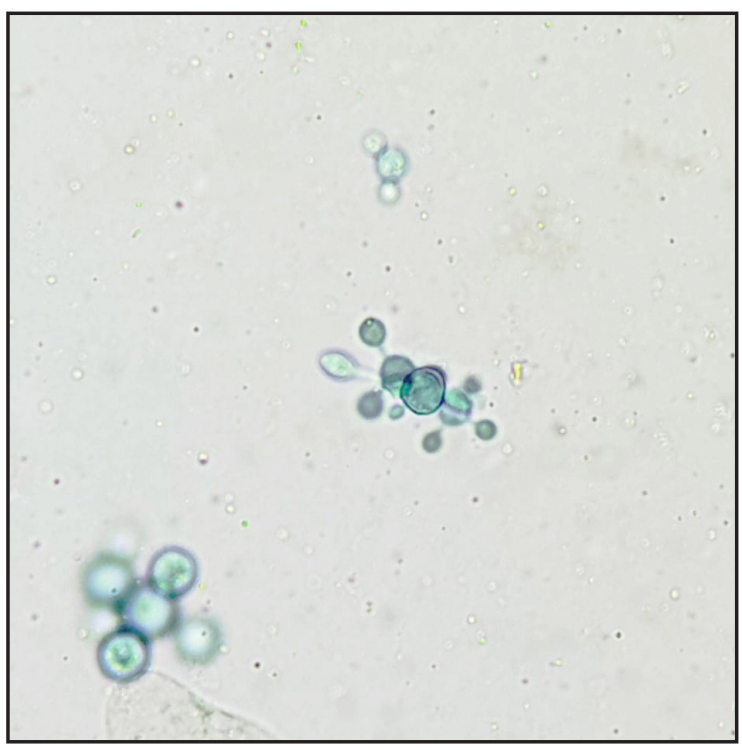

En el hemograma: hemoglobina 8,9 gr, glóbulos blancos 10.330. VSG: 18. Hepatograma y perfil renal normales. Na: 133, K: 4,4, Cl: 98.

Cortisol AM: 0,1ug/dl (valor normal 6,2-19,4), confirma Paracoccidioides $\mathrm{sp}$ en ambos materiales (Figura 6). BAAR negativo en esputo.

Figura 6. Cultivo. Paracoccidiodoides $s p$

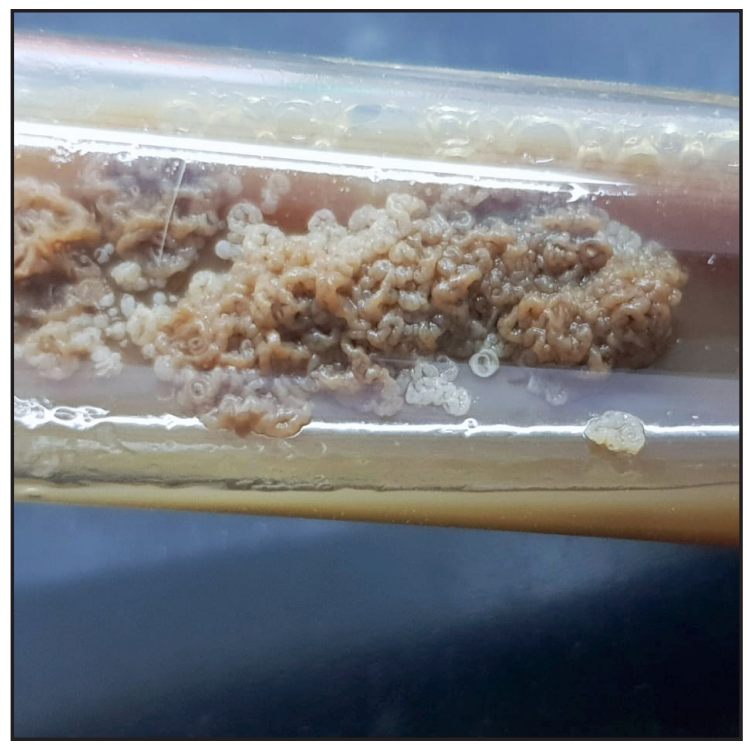

cortisol PM: 0,9 ug/dl (valor normal: 2,3 -11,9).

Radiografía de tórax: Infiltrado intersticial difuso a predominio de base pulmonar derecha y campo medio (Figura 7).

Figura 7. Radiografía, de tórax. Importante

compromiso por paracoccidioidomicosis

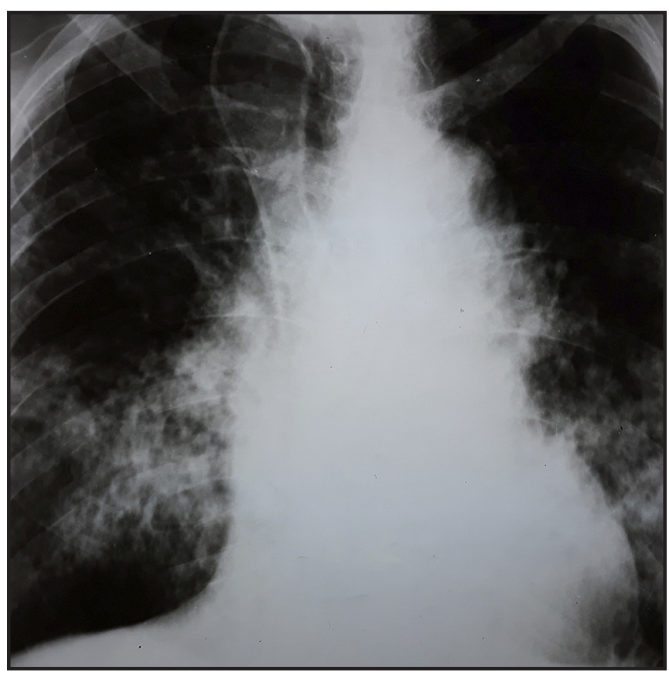


La resonancia magnética de abdomen informa aumento difuso de glándulas suprarrenales con predominio del lado derecho.

Con estos hallazgos se llega al diagnóstico de paracoccidioidomicosis crónica con afectación multifocal, interpretándose la insuficiencia suprarrenal como causada por la micosis.

El paciente es tratado, además de la sustitución hormonal, con anfotericina B, llegándose a 1,5 gramos, y luego itraconazol $200 \mathrm{mg} / \mathrm{dia}$, con mejoría de las lesiones cutáneas, del cuadro pulmonar y del estado general.

\section{COMENTARIOS}

La paracoccidioidomicosis, es una micosis profunda que presenta una distribución geográfica específica, circunscripta a América desde México hasta la Argentina. Presenta formas clínicas agudas, subagudas y crónicas, y ésta última compromiso unifocal y multifocal, siendo ésta la más frecuente $(5,6)$. El paciente pertenecía a este último grupo.

Esta micosis afecta mucosas, piel y órganos internos en forma aislada o múltiple. Puede comprometer cualquier órgano, pero los más frecuentemente pulmones, ganglios linfáticos, bazo, hígado, intestino, y glándulas suprarrenales $(3,4,6,7)$.

Las lesiones cutáneas de la paracoccidioidomicosis se observan aproximadamente en el $30 \%$ de los casos, siendo lesiones polimorfas, más frecuentemente ulcerosas con finas granulaciones. Generalmente facilitan el diagnóstico, al ser muy accesibles a los estudios micológicos y anatomopatológicos $(6,8)$. En éste caso en particular el agente fue aislado primeramente en las úlceras de miembros inferiores y este hallazgo orientó a la probable causa de la IS y del cuadro pulmonar, confirmado al aislar el agente en el esputo.

La confirmación que la IS es producida por el Paracoccidiodes se tiene al aislar el agente en el órgano, por punción o biopsia $(7,9,10)$. En este caso no fue realizado por no haber sido la sospecha inicial y posteriomente por la buena evolución. La resonancia magnética apoyó al diagnóstico clínico. También la ultrasonografía y la tomografía son recursos importantes $(7,11)$.
El signo dermatológico mas importante de la IS primaria es la hiperpigmentación, que afecta sobre todo a áreas de piel sujetas a presión, expuestas al sol, pliegues de flexión y mucosas; causada por la estimulación de los melanocitos por la ACTH y MSH aumentadas al disminuir la cortisona(1).

En el Brasil y otros países de América del Sur la paracoccidioidomicosis es una de las causas mas frecuentes de Addison. Entre 10 al 15\% de los pacientes con paracoccidioidomicosis presentan afectación suprarrenal clínicamente evidente y el $3 \%$ pueden desarrollar una crisis addisoniana (12).

Las suprarrenales son la cuarta en frecuencia de afectación por el Paracoccidioides, luego del tegumento, pulmones y ganglios, demostrando el tropismo de esa glándula por los microorganismos, debido a la elevada concentración de glucocorticoides, que produce una inmunosupresión local $(3,4)$.

El compromiso suprarrenal se observó en el $50 \%$ de autopsias de pacientes con paracoccidiodomicosis, sin síntomas de deficiencia suprarrenal. Para que presente síntomas debe ocurrir la destrucción de por lo menos 80 a $90 \%$ de la glándula. Excepcionalmente el compromiso de las suprarrenales puede ser única localización de la micosis o primera manifestación $(12,13)$.

\section{Conflicto de intereses y financiación}

Ningún conflicto y la financiación es propia.

\section{Agradecimientos}

- Dr. José Pereira

- Dr. Luis Celias

\section{REFERENCIAS BIBLIOGRAFICAS}

1. Arit W. Transtornos de la corteza suprarrenal. En: Longo D, Fauci A, Kasper D, Hauser S, Jameson J, Loscalzo J. Harrison. Principios de Medicina Interna.18 ed. Mèxico: Mc Graw Hill 2012, p.2940-2961.

2. Kalus A, Chien A, Olerud J. Diabetes mellitus y otras enfermedades endócrinas. En : Goldsmith L, Katz S, Gilchrest B, Paller A, Lefell D, Wolff K. Fitzpatrick. Dermatologìa en Medicina General. $8^{a}$ Ed. Buenos Aires: Panamericana 2012, p.1839-1868. 
3. Oelkers W. Adrenal insufficiency. New Eng J Med 1996; 16:1206-12.

4. Oñate J, Tobòn A, Restrepo A. Insuficiencia suprarrenal secundaria a paracoccidioidomicosis. Biomèdica 2002: 280-6.

5. Arenas R. Micología médica ilustrada. $4^{a}$ ed. México: Mc.Graw Hill 2011.

6. Marques S. Paracoccidioidomycosis: epidemiological, clinical, diagnostic and treatment up-dating. An Bras Dermatol. 2013; 88 (5): 700-11. 9.

7. Sanabria $C$, Alarcòn $M$, Alarcòn $I$, Jaimes $M$. Paracoccidiodomicosis . Una enfermedad multisitèmica. Acta Med Colomb 2018; 43: 111-114.

8. Aldama A. Dermatologia Tropical. Atlas y manejo. Asunciòn: Visualmente 2010.

9. Farina M, Espinola C, Yamanaka W, Bernal M, Villalba J. Paracoccidioidomicosis en la glándula suprarrenal. Reporte de un caso. Rev Chilena de Cirugía 2007; 59: 299-301.

10. Faiçal S, Borri ML, Hauache OM, Ajzen S. Addison's disease caused by Paracoccidioides brasiliensis: diagnosis by needle aspiration biopsy of the adrenal gland. AJR 1996;166:461-12.

11. Kawashima A,B, Sandler CM, Fishman EK, et al. Spectrum of $C T$ findings in nonmalignant disease of the adrenal gland. RadioGraphics 1998;18:393- 412.

12. Cermeño J, Cermeño J, Cova N, Pérez G. Función adrenocortical en pacientes con micosis sitémica. Invest clin. 2007; 48 (1): 341-8.

13. Marsiglia I, Pinto J. Adrenal cortical insufficiency associated with paracoccidioidomycosis (South American blastomycosis). Report of four patients. J Clin Endocrinol Metab 1966; 26:1109-11. 